\title{
Contraste de un modelo de envejecimiento exitoso derivado del modelo de Roy
}

\author{
María Amparo Kantún Marín*, José Moral de la Rubia**, Bertha Cecilia Salazar González* y Oscar Rosas Carrasco***
}

Recepción: 3 de mayo de 2016

Aceptación: 27 de febrero de 2017

*Universidad Autónoma del Carmen, México.

**Universidad Autónoma de Nuevo León, México.

${ }^{* * *}$ Instituto Nacional de Geriatría, México.

Correos electrónicos: m_kantun@hotmail.com;

jose_moral@hotmail.com; bceci195@hotmail.com;

oscarc.rosas@salud.gob.mx

Se agradecen los comentarios de los árbitros de la revista.

(c) $\$(\$$

\section{Introducción}

Se puede definir el envejecimiento exitoso como la adaptación a los cambios físicos, fisiológicos y sociales que se producen en el adulto mayor con el paso del tiempo (wHO, 1992). La percepción de la salud física, satisfacción con la vida y la sociabilidad se consideran indicadores del envejecimiento exitoso entendido como una respuesta de adaptación (Deng et al., 2010; Dogra y Stathoskostas, 2012; Ng et al., 2009). Lee et al. (2011) señalan que mantener la salud física y la funcionalidad psicosocial, contar con apoyo social e involucrarse en actividades de ocio placenteras y estimulantes son los cuatro factores que definen un proceso de envejecimiento exitoso.

\begin{abstract}
Resumen. Se formula y contrasta un modelo de envejecimiento exitoso derivado del modelo de adaptación de Roy. Se analizó una muestra no probabilística de 255 adultos mayores de seis centros sociales y un centro médico de consulta externa en Nuevo León, México. Se usó un modelamiento de ecuaciones estructu- En modelo final los estímulos focales peatonal inmediato y el estímulo contextual de síntomas depresivos incidieron sobre el afrontamiento, que a su vez predijo el envejecimiento exitoso. El modelo tuvo buen ajuste al añadirse dos correlaciones entre residuos de medida de afrontamiento y envejecimiento exitoso. Los resultados obtenidos sugieren que el afrontamiento es un indicador directo del envejecimiento activo.
\end{abstract}

Palabras clave: envejecimiento, depresión, satisfacción con la vida, afrontamiento, función cognitiva.

\author{
Contrast of a Successful Aging Model \\ Derived from the Roy's Model \\ Abstract. The objective of the study was to \\ formulate and contrast a successful aging model \\ derived from the Roy's adaptation model. A \\ nonrandom sample of 255 adults from six social \\ centers and one clinical center from Nuevo \\ Leon, Mexico was analyzed. A structural equa- \\ tion modeling was used for data analysis. In the \\ final model, the focal stimuli of self-perception \\ of aging and immediate pedestrian environ- \\ ment and the contextual stimuli of depressive \\ symptoms had an impact on coping. In turn, \\ coping predicted successful aging. The model \\ had a good adjustment to the data by adding two \\ correlations between the residuals of the mea- \\ surement of coping and successful aging. The \\ results suggest that coping is a direct indicator of \\ active aging.
}

Keywords: aging, depression, life satisfaction, coping, cognitive function.
De acuerdo con el modelo de adaptación de Roy (2009; Roy y Andrews, 1999), las personas son sistemas adaptativos que operan mediante entradas, procesos y salidas. Las entradas al sistema constituyen los estímulos que activan los procesos de afrontamiento para generar las salidas, lo que se conoce como conductas o respuestas. Se puede definir estímulo como cualquier fenómeno interno o externo a la persona que provoca una conducta o respuesta. El modelo propone tres tipos de estímulos: focal, contextual y residual (Roy, 2009; Roy y Andrews, 1999). El estímulo focal confronta inmediatamente a la persona en un momento determinado y demanda toda su atención o energía. Este estímulo es el más cercano a la conciencia de la persona. Un ejemplo de 
estímulo focal sería percibir la disminución en las capacidades motoras, sensoriales e intelectuales que puede ser evaluada por el Cuestionario de percepción de envejecimiento de Barker et al. (2007). Otro ejemplo sería la movilidad en el entorno inmediato, como el entorno peatonal del vecindario, que puede ser evaluada por la escala abreviada de Cerin et al. (2006). Los estímulos contextuales presentes en un momento dado contribuyen a los efectos del estímulo focal e influyen en la conducta o respuesta, pero sin convertirse en centro de atención. Un ejemplo de estímulos contextuales sería la actividad física de caminar que puede ser medida por medio de un podómetro. Otro ejemplo sería un estado de ánimo depresivo manifiesto o consciente que distorsiona la percepción hacia una mayor disminución de las propias capacidades, el cual puede ser evaluado por la escala de Herrero y Gracia (2007). Los estímulos residuales son factores cuyos efectos no están en la conciencia de la persona, pero influyen en la respuesta. Cuando acceden a la conciencia dejan de ser residuales y pasan a ser contextuales. Un ejemplo sería un estado de ánimo depresivo enmascarado o inconsciente que distorsiona la percepción hacia una mayor disminución de capacidades (Miodek et al., 2007). Usualmente, los estímulos residuales se omiten al derivar propuestas teóricas o de trabajo desde el modelo de adaptación de Roy, debido a que es complicado evaluar aspectos inconscientes y detectar el estado latente de un fenómeno psíquico patológico. Al respecto Roy y Andrews (1999) señalan que los estímulos focales involucran las creencias y actitudes sobre los que la persona no tiene conciencia, por lo que no se pueden investigar. En este artículo al medirse la depresión por medio de una escala de autorreporte que no diferencia entre depresión latente y manifiesta, se consideró a la depresión como un estímulo contextual.

Los estímulos activan los procesos de afrontamiento, los cuales se pueden definir como las formas innatas o adquiridas de responder al entorno cambiante. Dentro de los procesos de afrontamiento para explicar el envejecimiento exitoso se puede incluir la capacidad funcional, los factores intrapsíquicos y la función cognitiva (Flood, 2005). La capacidad funcional comprende la forma en que una persona responde conscientemente a la acumulación de cambios funcionales que se producen como consecuencia del paso del tiempo, así como el uso de su capacidad de elección para decidir realizar o no las actividades cotidianas. La capacidad funcional puede ser evaluada usando los dos primeros ítems del Inventario de envejecimiento exitoso de Troutman et al. (2011). Un segundo tipo de proceso de afrontamiento son los factores intrapsíquicos que se refieren a las características innatas y rasgos perdurables del carácter de una persona que pueden aumentar o alterar su habilidad para adaptarse al cambio y resolver problemas. Ejemplos de factores intrapsíquicos que facilitan la adaptación serían el optimismo, la creatividad, la afectividad positiva y el control personal (Conversano et al., 2010). El Inventario de Troutman et al. (2011) agrupa dentro de un factor a siete ítems que evalúan estos rasgos. Un tercer tipo de proceso de afrontamiento es la función cognitiva. En el adulto mayor se refiere a la conservación de las capacidades intelectuales o cognitivas (Roy, 2009; Roy y Andrews, 1999), y puede ser evaluada por medio del examen cognitivo de Mathuranath et al. (2000).

De acuerdo con el modelo de adaptación de Roy, las respuestas de afrontamiento exitoso (adaptación) se podrían dar bajo cuatro modos: a) fisiológico, $b$ ) autoconcepto, $c$ ) interdependencia y d) de función de rol (Roy, 2009; Roy y Andrews, 1999). El modo fisiológico está asociado con los procesos físicos y químicos involucrados en la función y actividad de los organismos vivos. Con este modo adaptativo se hace referencia a la percepción de salud en su dimensión física, como uno de los factores del cuestionario de Ware Jr et al. (1996). El modo de autoconcepto se refiere al conjunto de creencias y sentimientos que la persona posee acerca de sí mismo en un momento dado. Un autoconcepto positivo es un factor promotor de envejecimiento exitoso y se refleja en sentimientos de satisfacción con la propia persona y con los logros personales. Desde esta manifestación se puede evaluar usando el Índice de satisfacción con la vida para la tercera edad de Barrett y Murk (2006). El modo de interdependencia es el modo de dar y recibir amor, respeto y valor en las relaciones interpersonales. Este tercer modo adaptativo se puede evaluar con la escala de sociabilidad de Rubio y Aleixandre (1999). Finalmente estaría el modo de rol, respuesta que se refiere al papel que ocupa el individuo en la sociedad de acuerdo con su etapa de desarrollo. En el adulto mayor la necesidad subyacente en el modo de función de rol es mantener la integración y funcionalidad sociales (Durán et al., 2008), por lo que el modo de rol, reducido a integración y funcionalidad sociales del adulto mayor se podría evaluar a través de la escala de sociabilidad de Rubio y Aleixandre (1999).

El objetivo del artículo es formular y contrastar un modelo de envejecimiento exitoso derivado del modelo de adaptación de Roy, el cual propone que los factores focales y contextuales son predictores de los procesos de afrontamiento, y los procesos de afrontamiento son predictores del envejecimiento exitoso (respuesta de adaptación) cuando los factores de estímulos contextuales y focales están interrelacionados. Los factores contextuales afectan a los focales y en ciertos momentos pueden pasar a ser focales.

En este caso, los estímulos focales fueron representados por la autopercepción del envejecimiento y las características 
del entorno inmediato (ambiente peatonal); los estímulos contextuales fueron representados por la actividad física de caminar y los síntomas de depresión. Siguiendo las indicaciones de Roy y Andrews (1999), los estímulos residuales se omitieron al ser difíciles de operativizar y de diferenciar de los estímulos contextuales. El proceso de afrontamiento fue representado por la función cognitiva, la capacidad funcional y los factores intrapsíquicos de creatividad, baja negatividad, afectividad positiva y control personal. Las respuestas adaptativas para el envejecimiento exitoso se representaron a través del modo fisiológico con la percepción de la salud física, del modo de autoconcepto con la satisfacción con la vida y del modo de interdependencia y de rol con la sociabilidadintegración social. Así, el modo de rol de la respuesta adaptativa se incluyó en la medición del modo de interdependencia como integración social (figura 1).

De acuerdo con el modelo de Roy (Beard y Petitot, 2010; Chodosh et al., 2007; Kleinspehn et al., 2008; Roy, 2009; Roy y Andrews, 1999), se formula que los estímulos focales (indicados por el entorno peatonal inmediato y la autopercepción del envejecimiento) y los estímulos contextuales (indicados por la actividad física de caminar y los síntomas de depresión) actúen directamente sobre los procesos de afrontamiento (indicados por la capacidad funcional, los factores intrapsíquicos de positividad y la función cognitiva) al estar relacionados los dos factores de estímulos, ya sea que los estímulos contextuales afecten directamente a los estímulos focales o que estímulos focales y contextuales correlacionen. Además, se postula que los procesos de afrontamiento impacten sobre el envejecimiento exitoso, indicado este último por la percepción de salud física (modo fisiológico), satisfacción con la vida (autoconcepto) y sociabilidad-integración social (interdependencia y rol) (figura 1).

\section{Método}

\section{1. Participantes}

Se realizó un muestreo no probabilístico. Se recolectó una muestra incidental de 255 adultos mayores pertenecientes a seis centros de Desarrollo Integral de la Familia de Nuevo León (DIF-NL) y un centro médico de consulta externa de la Universidad Autónoma de Nuevo León (UANL). Los criterios de inclusión fueron: a) tener 60 años cumplidos, $b$ ) capacidad de deambular sin ayuda y $c$ ) dar el consentimiento informado. Los criterios de exclusión fueron: a) incapacidad o dificultades para leer, $b$ ) comprender y $c$ ) llevar el registro de su deambulación por medio de un podómetro. Los criterios de eliminación fueron: a) no completar el registro semanal de pasos y $b$ ) dejar más de $10 \%$ de las preguntas de una escala o cuestionario sin contestar.

De los 255 participantes, $51 \%$ procedía del DIF-NL y $49 \%$ de la Unidad de Servicios Médicos de la unant. De los participantes, $82.7 \%$ fue mujer y $17.3 \%$ hombre. La edad varió de 60 a 100 años con una media de 69.27 años $(D E=6.51)$. Entre 60 y 69 años se encontró a $60 \%$ de los participantes, entre 70 y 79 a $36 \%$ y entre 80 y 100 a $4 \%$ restante. La

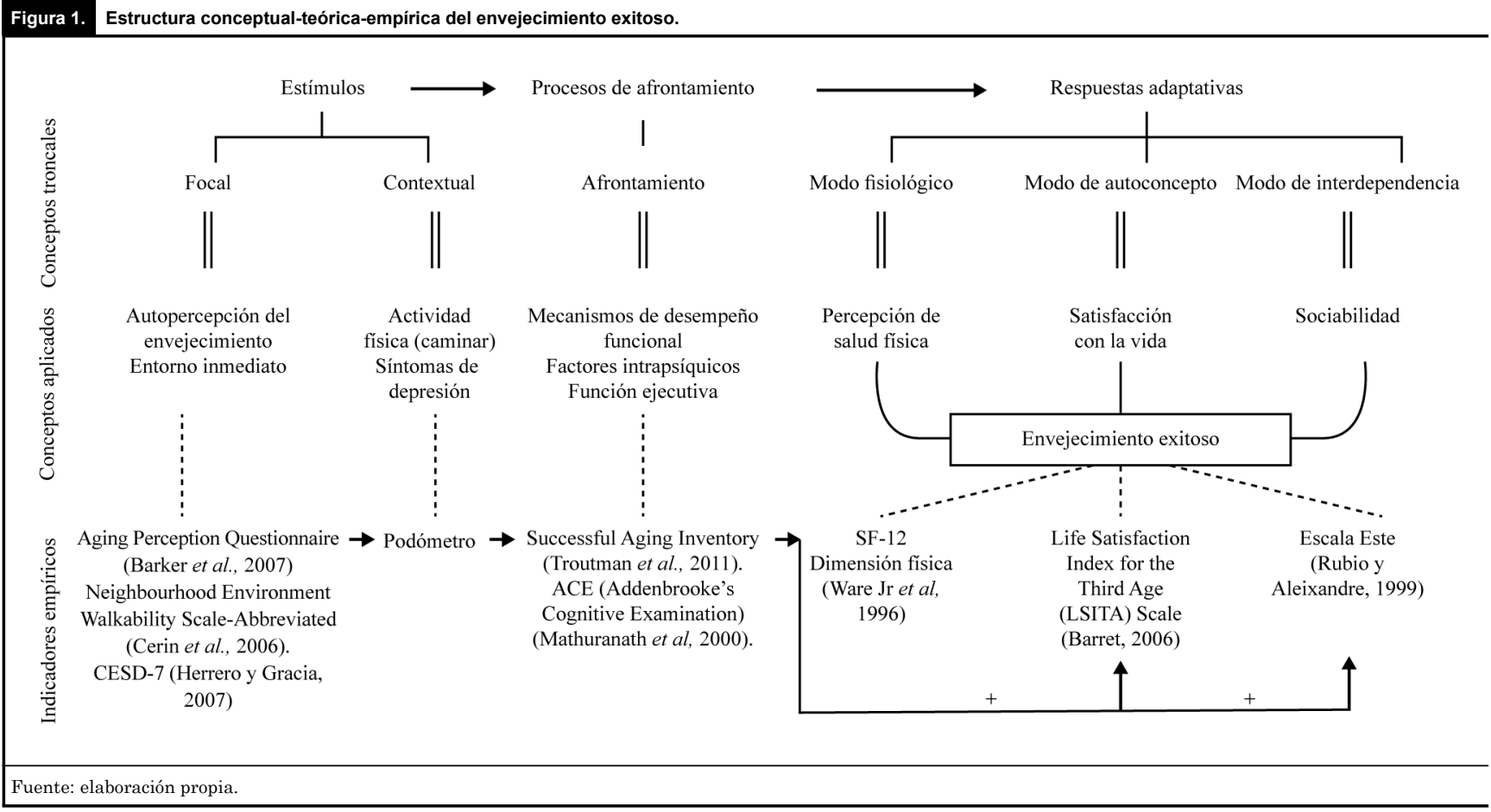


media de años de escolaridad fue de $7.27(D E=4.80)$. En cuanto a escolaridad $32.9 \%$ tuvo primaria trunca (menos de 6 años), $28.6 \%$ de al menos primaria terminada (menos de 9 años), $19.6 \%$ de al menos secundaria terminada (menos de 11 años), $7.5 \%$ de media superior terminada (11 o 12 años) y $11.4 \%$ de estudios universitarios (más de 12 años). Respecto al estado civil, $49 \%$ estaba casado, $38 \%$ viudo, $7 \%$ soltero, $4 \%$ separado y $2 \%$ divorciado. En relación con el estatus laboral, $66.6 \%$ señaló dedicarse al hogar, $22.6 \%$ está jubilado y $10.8 \%$ labora actualmente. En cuanto a salud $92.9 \%$ de los adultos mayores dijo tener uno o más problemas de salud y $7.1 \%$ ninguno, $89.4 \%$ de los participantes mencionaron que toman medicamentos y $10.6 \%$ no.

\section{2. Variables e instrumentos de medida}

Se aplicó una cédula de datos sociodemográficos (sexo, edad, escolaridad, estado civil y estatus laboral) y problemas de salud (enfermedades y uso de medicamentos); asimismo, se evaluaron los diez indicadores de las cuatro variables latentes (estímulos focales, estímulos contextuales, afrontamiento y envejecimiento exitoso) por medio de ocho escalas de autorreporte y un podómetro. Para dos de los indicadores (las variables manifiestas capacidad funcional y factores intrapsíquicos) se emplearon distintos ítems de una misma escala (el SAI). Los instrumentos de medida se administraron en el siguiente orden fijo:

a) La autopercepción del envejecimiento (indicador de la variable latente de estímulos focales) se evaluó a través del Cuestionario de percepción de envejecimiento (Aging Perception Questionnaire, APQ) de Barker et al. (2007). Se empleó la puntuación total: una mayor puntuación refleja mayor conciencia de envejecimiento que motiva una actitud activa y responsable ante las progresivas pérdidas y creciente dependencia de los demás. En esta muestra sus 65 ítems tuvieron una consistencia interna alta $(\alpha=0.82)$.

b) El entorno inmediato (indicador de la variable latente de estímulos focales) se evaluó con la Escala breve del ambiente peatonal del vecindario (Neighbourhood Environment Walkability Scale-Abbreviated, NEwS-A) de Cerin $e t$ al. (2006). Se empleó la puntuación total. La escala evalúa la infraestructura del vecindario que se cree promueve la caminata para transportarse y practicar actividades de ocio. Una mayor puntuación refleja mayor posibilidad de caminar. En esta muestra sus 47 ítems tuvieron una consistencia interna alta $(\alpha=0.89)$.

c) Los síntomas de depresión (indicador de la variable latente de estímulos contextuales) fueron evaluados con la Escala de Depresión del Centro de Estudios Epidemiológicos en su forma abreviada (CESD-7) (Herrero y Gracia,
2007; Salinas et al., 2014). Se empleó la puntuación total. Se puntuó 0 a la opción de respuesta la mayor parte del tiempo o todo el tiempo, 1 a la opción ocasionalmente, 2 a la opción alguna veces y 3 a la opción raramente o nunca. Como las demás escalas, se puntuó en sentido positivo, por lo que una mayor puntuación refleja menos síntomas de depresión. En esta muestra sus siete ítems tuvieron una consistencia interna aceptable $(\alpha=0.75)$.

d) La capacidad funcional (indicador de la variable latente de procesos de afrontamiento) se evaluó con los dos primeros ítems sobre desempeño funcional del Inventario de envejecimiento exitoso (Successful Aging Inventory, SAI) de Troutman et al. (2011). Estos dos ítems de desempeño funcional mostraron una consistencia interna cuestionable $(\alpha=0.66)$. Los factores intrapsíquicos (indicadores de la variable latente de procesos de afrontamiento) se evaluaron con los siete ítems del SAI sobre capacidades personales para adaptarse a los cambios y resolver problemas, creatividad, baja negatividad, afectividad positiva y control personal. La consistencia interna de estos siete ítems fue alta $(\alpha=0.82)$. Debe señalarse que se aplicaron sólo nueve de los 20 ítems del sai para evaluar dos de sus cinco factores (capacidad funcional y factores intrapsíquicos). Para superar el problema de consistencia interna cuestionable del factor de capacidad funcional, se exploró la estructura factorial de los nueve ítems. Por el análisis paralelo de Horn el número de factores fue uno. Al ser extraído el factor por factorización de ejes principales, se explicó 40.2\% de la varianza total. Las saturaciones de los nueve ítems en el factor fueron altas, pues varió de 0.55 a 0.74 con una media de 0.63. Por su contenido, se denominó capacidad funcional y positividad para afrontar los cambios del envejecimiento. La consistencia interna de los nueve ítems fue alta $(\alpha=0.85)$. En los análisis se usó esta variable de capacidad funcional/ positividad definida por la suma simple de los nueve ítems.

e) La función cognitiva (indicador de la variable latente de procesos de afrontamiento) se evaluó a través de la orientación en tiempo y espacio, concentración/atención, memoria, fluencia verbal, lenguaje y habilidades visuoespaciales, usando el Examen Cognitivo de Addenbrooke (ACE) de Mathuranath et al. (2000). Se empleó la puntuación total. Mayor puntuación refleja mayor funcionalidad. En esta muestra sus 40 ítems tuvieron una consistencia interna alta $(\alpha=0.80)$.

f) La percepción de la salud física (indicador de la variable latente de envejecimiento exitoso) se evalúo con el Cuestionario de percepción de salud (12-Item Short-Form Health Survey, SF-12) de Ware Jr et al. (1996), que utilizó el factor de salud física. Se empleó la puntuación del factor. Mayor puntuación refleja percepción de mayor salud. En esta muestra sus cinco ítems tuvieron una consistencia interna alta $(\alpha=0.80)$. 
g) La manifestación del modo de autoconcepto de la respuesta adaptativa de envejecimiento exitoso se evaluó con el Índice de satisfacción con la vida de la tercera edadversión breve (LSITA-SF) de Barrett y Murk (2006). Se empleó la puntuación total. Mayor puntuación refleja mayor satisfacción con la vida. En esta muestra sus 12 ítems tuvieron una consistencia interna aceptable $(\alpha=0.77)$, como se reporta en el estudio de Kantún-Marín et al. (2013).

h) La sociabilidad es la relación de afectividad que el adulto mayor sostiene con otras personas, la expresión de sentirse valorado y apoyado por otros y la expresión de sentir lo mismo para los demás. Es el tercer indicador de la variable latente de envejecimiento exitoso. Se midió con la Escala Este de Rubio y Aleixandre (1999) que evalúa la autopercepción de soledad, apoyo social recibido y nivel de satisfacción con los contactos sociales. Se empleó la puntuación total. Mayor puntuación refleja más sociabilidad. En esta muestra sus 33 ítems tuvieron una consistencia interna alta $(\alpha=0.87)$. Bajo esta variable también se incluyó el modo de rol (integración y funcionalidad sociales) o cuarto indicador del envejecimiento exitoso.

i) La actividad física diaria de caminar (indicador de la variable latente de estímulos contextuales) se evaluó mediante el uso de un podómetro. El intervalo de tiempo de registro continuo durante las horas de vigilia fue de seis días. Se usó

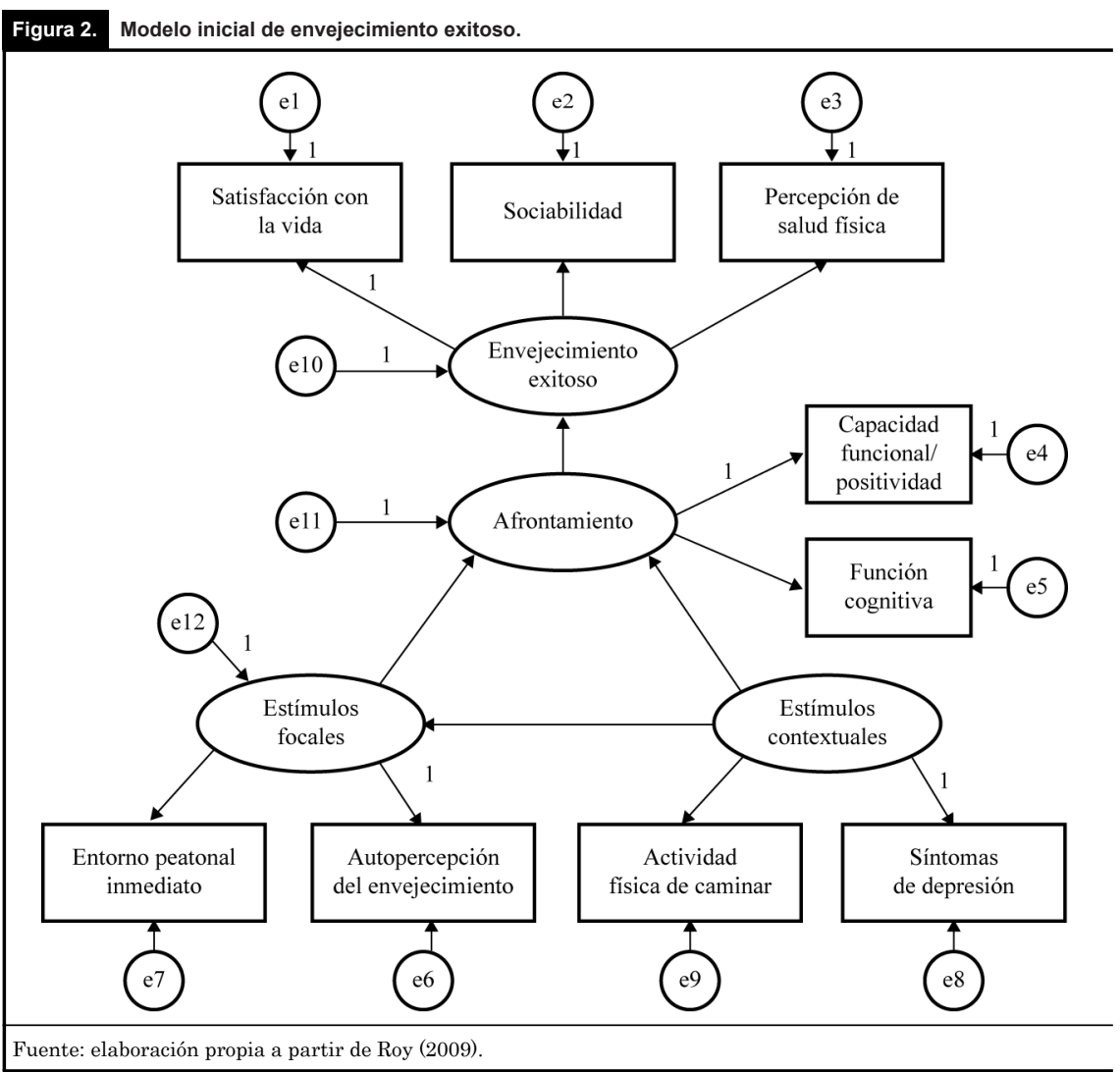

el podómetro electrónico Yamax-Digiwalker sw-701 (Crouter et al., 2003).

\section{3. Procedimiento}

La investigadora principal se entrevistó con el personal responsable de los programas para adultos mayores del DIF-NL y de los servicios médicos de la UANL. Explicó el propósito del estudio y solicitó la autorización para llevarlo a cabo. Se seleccionaron al azar 6 de los 28 centros del DIF-NL. Las entrevistas a los participantes se efectuaron en los sitios y horarios autorizados por ayudantes de investigación capacitados. Después de rellenar los cuestionarios, los participantes recibieron un podómetro para contar los pasos cuando caminan, corren o trotan en el transcurso de las horas de vigilia durante seis días. Tras seis días de uso del podómetro, los participantes fueron citados en el centro en el cual fueron entrevistados para la recogida del aparato. Los datos fueron recolectados por la investigadora principal y por asistentes de investigación entrenados para tal fin entre enero y abril de 2012. El estudio se realizó conforme con las normas éticas de American Psychological Association (2002), se solicitó el consentimiento informado para garantizar la confidencialidad de la información y no generar ningún daño o perjuicio. El estudio fue aprobado por el Comité de Ética Institucional.

Para analizar los datos, se utilizó modelamiento de ecuaciones estructurales por el método de Máxima Verosimilitud (ML) a través del Amos versión 16.0 (Arbukle, 2007). Los valores perdidos fueron sustituidos por la media.

\section{Resultados}

Se partió del modelo derivado para el envejecimiento exitoso del modelo de adaptación de Roy (2009) (figura 2). Debido a que la solución no fue admisible, se hicieron cambios sobre las variables latentes de estímulos focales y contextuales para finalmente eliminarlas y conservar sus indicadores como variables manifiestas exógenas correlacionadas (dos de estímulos focales y uno de contextuales) (figuras 3 y 4). A continuación, para mejorar la bondad de ajuste se liberaron dos parámetros del modelo (dos correlaciones entre residuos de medida) tras revisar los 
índices de mejora del ajuste, con lo que se logró un modelo final con buen ajuste a los datos (figura 5).

El modelo de envejecimiento exitoso propuesto (inicial) quedó integrado por cinco variables latentes cuyos modelos de medida se presentan en la tabla 1. El modelo estructural (relación entre las cinco variables latentes) quedó especificado del siguiente modo: la variable endógena (final) fue el envejecimiento exitoso y fue pronosticada por el afrontamiento. A su vez, el afrontamiento como variable endógena (intermedia) fue predicha por los estímulos contextuales y contextuales. Los estímulos focales también se especificaron como una variable endógena y fueron predichos por los estímulos contextuales. Estos últimos quedaron como una variable exógena (no predicha). Todos los residuos (de medida y estructurales) se especificaron como independientes (figura 2).

Al elaborar la estimación del modelo, no se obtuvo una solución. Apareció un error motivado por la predicción de los estímulos focales por los residuales. Este error no desapareció al especificar a los estímulos focales y contextuales como dos variables exógenas correlacionadas. Una vez que ambas variables exógenas se especificaron como independientes se logró obtener una solución, pero ésta fue no admisible (figura 3). La varianza de un residuo resultó negativa $(\operatorname{var}[\mathrm{e} 11]=-9.88, E E=$ 17.43, $z=-0.57, p=0.57$ ), el coeficiente $\beta$ de la predicción del afrontamiento por los estímulos focales fue mayor a $1(\beta=1.08)$ y el porcentaje de varianza explicada del factor de afrontamiento fue mayor a 100\% (149.6\%). Se observó que la actividad física no fue un indicador significativo del factor de estímulos contextuales $(B=64.70, E E=143.90, z=0.45, p=0.65, \beta=$ $0.05)$ y que este factor no predijo de forma significativa el afrontamiento $(B=0.16, E E=0.33, z=0.48, p=0.63, \beta=$ 0.58) (figura 3).

Desde esta consideración se eliminó el factor de estímulos contextuales, pero se conservó uno de sus indicadores (síntomas de depresión) como la variable manifiesta exógena, lo que

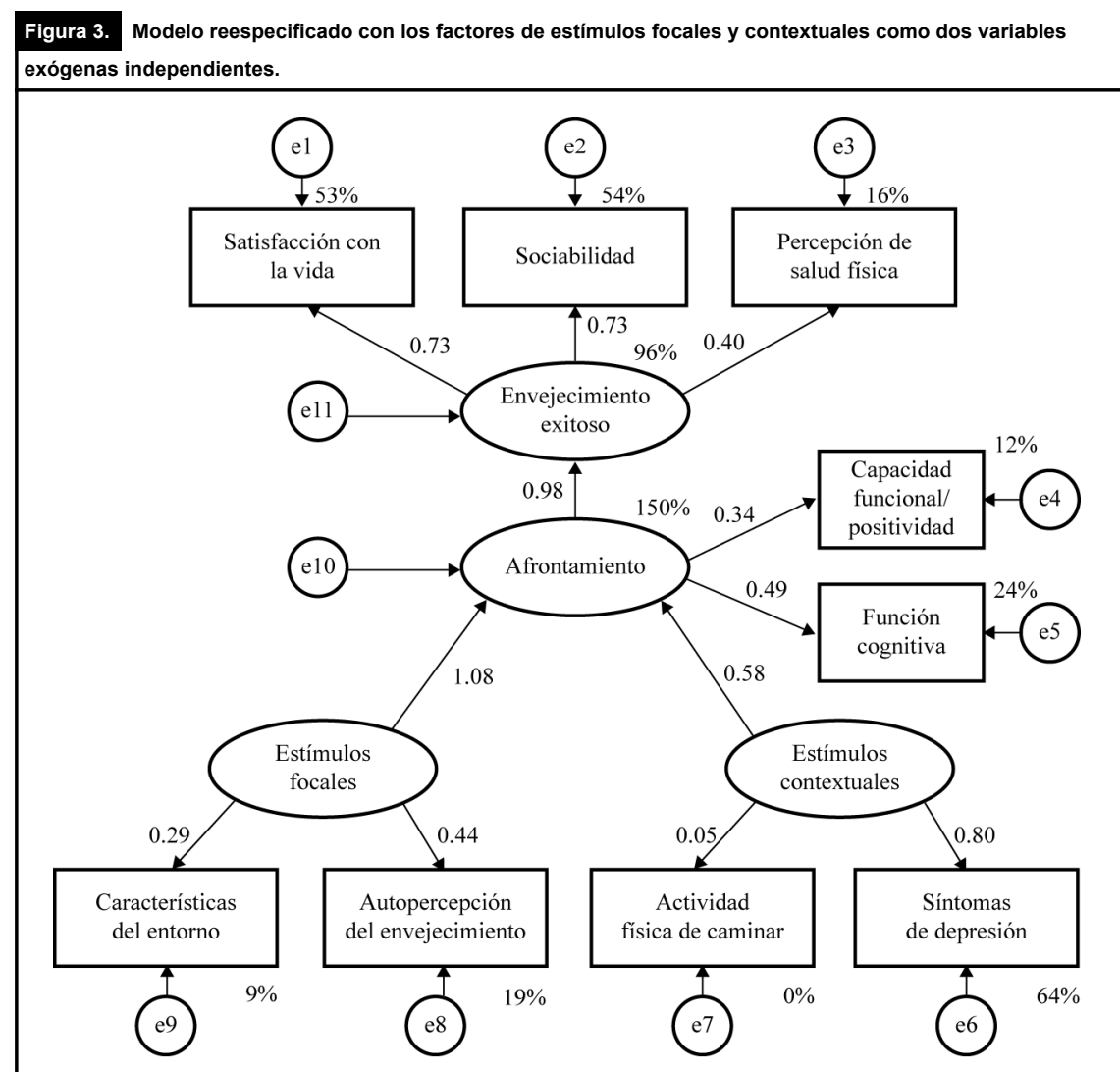

Nota: índices de ajuste: $\chi^{2}(24, N=255)=95.62, p<0.01 ; \mathrm{NFI}=0.79 ; \mathrm{CFI}=0.83 ; \mathrm{SRMR}=0.09 ; \mathrm{RMSEA}=0.11 ;$ $\mathrm{GFI}=0.93$ y $\mathrm{AGFI}=0.86$ Fuente: elaboración propia.

\begin{tabular}{|c|c|c|c|}
\hline \multicolumn{2}{|c|}{ Variable latentes } & \multirow{2}{*}{$\begin{array}{c}\text { Indicadores } \\
\text { (variables manifiestas) }\end{array}$} & \multirow[t]{2}{*}{ Instrumentos de medida } \\
\hline Tipo & Nombre & & \\
\hline \multirow{3}{*}{ Endógena final } & \multirow{3}{*}{ Envejecimiento exitoso } & Satisfacción con la vida (modo de autoconcepto) & LSITA-SF de Barrett y Murk (2006) \\
\hline & & Sociabilidad (modo de interdependencia y de rol) & Este de Rubio y Aleixandre (1999) \\
\hline & & Percepción de la salud física (modo fisiológico) & SF-12 de Ware Jr et al. (1996) \\
\hline \multirow{3}{*}{ Endógena intermedia } & \multirow{3}{*}{ Afrontamiento } & Capacidad funcional & SAI de Troutman et al. (2011) \\
\hline & & Positividad (factores intrapsíquicos) & \\
\hline & & Función cognitiva & ACE de Mathuranath et al. (2000) \\
\hline \multirow{2}{*}{ Endógena inicial } & \multirow{2}{*}{ Estímulos focales } & Entorno inmediato reducido al entorno peatonal del vecindario & NEWS-A de Cerin et al. (2006) \\
\hline & & Autopercepción del envejecimiento & APQ de Barker et al. (2007) \\
\hline \multirow{2}{*}{ Exógena } & \multirow{2}{*}{ Estímulos contextuales } & Actividad física de caminar & Pasos diarios medidos por un podómetro \\
\hline & & Síntomas de depresión & CESD-7 de Herrero y Gracia (2007) \\
\hline
\end{tabular}


definió un nuevo modelo (figura 4). Al estimar este nuevo modelo resultó la varianza de un residuo negativa (var [e11] $=-7.39, E E=10.43, z=-0.71, p=0.48)$, el coeficiente $\beta$ de
Modelo reespecificado en el que los estímulos contextuales se reducen a síntomas de depresión.

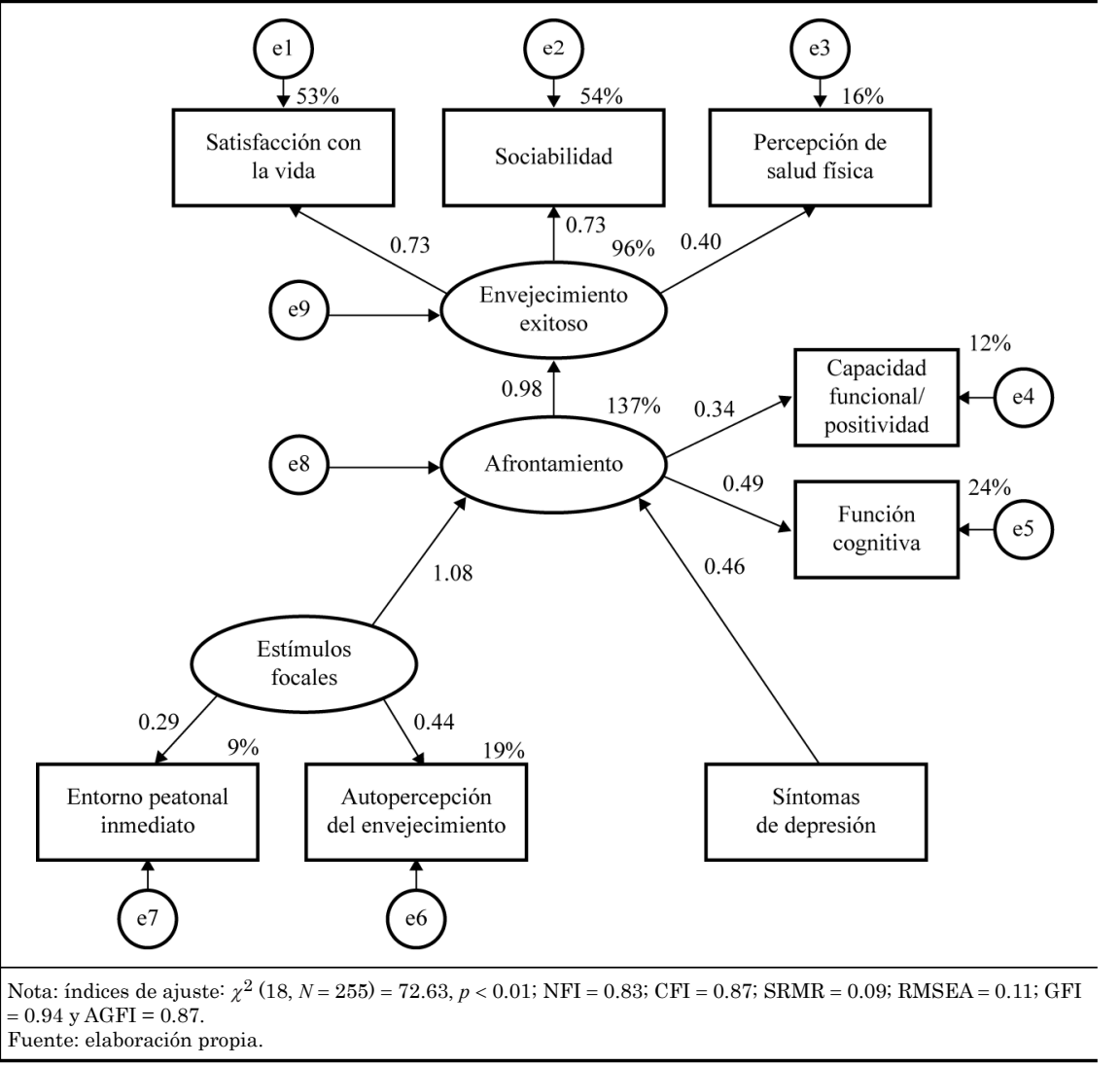

Cuadro 1.

Índices de ajuste del modelo con los estímulos como tres variables manifiestas correlacionadas.

\begin{tabular}{|c|c|c|c|c|c|}
\hline \multirow{2}{*}{$\begin{array}{l}\text { Índices } \\
\text { de ajuste }\end{array}$} & \multicolumn{2}{|c|}{ Interpretación } & \multicolumn{3}{|c|}{ Modelos con soluciones admisibles } \\
\hline & Bueno & Malo & $\begin{array}{c}\text { Con todos } \\
\text { los residuos } \\
\text { independientes }\end{array}$ & $\begin{array}{c}\text { Con una correlación } \\
\text { entre residuos de } \\
\text { medida }\end{array}$ & $\begin{array}{l}\text { Con dos correlaciones } \\
\text { entre residuos de me- } \\
\text { dida (modelo final) }\end{array}$ \\
\hline$\chi^{2}$ & & & 47.89 & 43.03 & 20.40 \\
\hline$g l$ & & & 16 & 15 & 14 \\
\hline$p$ & $>0.05$ & $<0.01$ & $<0.01$ & $<0.01$ & 0.12 \\
\hline$\chi^{2} / g l$ & $\leq 2$ & $\geq 3$ & 2.99 & 2.87 & 1.46 \\
\hline$p_{B-S}$ & $>0.05$ & $<0.01$ & $<0.01$ & $<0.01$ & 0.21 \\
\hline GFI & $\geq 0.95$ & $<0.90$ & 0.96 & 0.96 & 0.98 \\
\hline AGFI & $\geq 0.90$ & $<0.80$ & 0.90 & 0.90 & 0.95 \\
\hline NFI & $\geq 0.90$ & $<0.80$ & 0.90 & 0.90 & 0.95 \\
\hline NNFI & $\geq 0.90$ & $<0.80$ & 0.86 & 0.87 & 0.97 \\
\hline RMSEA & $\leq 0.05$ & $>0.09$ & 0.09 & 0.09 & 0.04 \\
\hline SRMR & $\leq 0.05$ & $>0.09$ & 0.05 & 0.05 & 0.04 \\
\hline
\end{tabular}

Nota: índices de ajuste: $\chi^{2}=$ estadístico chi-cuadrado de Pearson, $g l=$ grados de libertad del estadístico chi-cuadrado, $p=$ probabilidad del estadístico chi-cuadrado, $\chi^{2} / g l=$ cociente entre el estadístico chi-cuadrado y sus grados de libertad, $p_{B-S}=$ probabilidad de muestreo repetitivo de Bollen-Stine con la extracción de 2000 muestras, GFI = índice de bondad de ajuste de Jöreskog y Sörbom, AGFI = índice de bondad de ajuste corregido de Jöreskog y Sörbom, NFI = índice normado de ajuste de Bentler y Bonett, NNFI = índice no normado de ajuste de Bentler y Bonett, RMSEA = error de aproximación cuadrático medio de Steiger y Lind y SRMR = error estandarizado cuadrático medio de Jöreskog y Sörbom. Primera modificación = correlación entre los residuos e1 y e4. Segunda modificación = correlación entre los residuos e3 y e4.

Fuente: elaboración propia. la predicción del afrontamiento por los estímulos focales fue mayor a $1(\beta=1.08)$ y el porcentaje de varianza explicada del factor de afrontamiento fue mayor a $100 \%$ (137\%), que resulta nuevamente una solución inadmisible (figura 4). Al considerar que el coeficiente estructural estandarizado del factor de estímulos focales fue mayor a $1(\beta=1.08)$, la autopercepción del envejecimiento fue un indicador débil de dicho factor $(9 \%$ de la varianza explicada) y el número de indicadores del factor fue reducido (figura 4). Se optó por eliminarlo y dejar a sus dos indicadores (autopercepción del envejecimiento y entorno peatonal inmediato) como variables manifiestas exógenas (figura 5), como previamente se hizo con el factor de estímulos contextuales.

Este nuevo modelo proporcionó una solución admisible y un ajuste adecuado (cuadro 1). El valor estandarizado de la curtosis multivariada de Mardia fue 7.62, lo que mostró desviación de la normalidad multivariada (valor esperado entre -2 y 2). Al incumplirse el supuesto de normalidad multivariada, se usaron procedimientos de muestreo repetitivo para complementar el contraste de la bondad de ajuste del modelo (probabilidad de Bollen-Stine) y de la significación de parámetros (método de Percentiles Corregidos de Sesgo [PCS]), considerando que $\mathrm{ML}$ es un método robusto ante el incumplimiento de normalidad multivariada. Por la probabilidad de Bollen-Stine se rechazó la bondad de ajuste (cuadro 1).

$\mathrm{Al}$ especificarse una correlación entre los residuos e1 y e4 mejoró significativamente el ajuste $\left(\Delta \chi^{2}[1]=4.87, p=0.03\right)$, pero la bondad de ajuste se siguió rechazando por la probabilidad de BollenStine (cuadro 1). Al especificarse una segunda correlación entre los residuos e3 y e4, mejoró significativamente el ajuste en comparación con la modificación previa $\left(\Delta \chi^{2}[1]=22.63, p<0.01\right)$ y el ajuste fue bueno por los nueve índices contemplados, incluyendo la probabilidad de Bollen-Stine (cuadro 1). Como en los dos modelos previos todos 
los parámetros fueron significativos por ML y PCs (figura 5 y cuadro 2).

En este último modelo el tamaño de efecto directo de los síntomas de depresión sobre el afrontamiento $(\beta=0.46$, IC 95\%: $0.33,0.58, p<0.001$ por PCS) y el indirecto sobre el envejecimiento exitoso ( $\beta=0.41$, IC $95 \%$ : 0.30, 0.53, $p<0.001$ por PCS) fueron medianos. También el tamaño de efecto directo de la autopercepción del envejecimiento sobre el afrontamiento $(\beta$ $=0.45$, IC 95\%: 0.32, 0.57, $p<0.001$ por PCS) y el indirecto sobre el envejecimiento exitoso $(\beta=0.40$, IC $95 \%$ : $0.30,0.50, p$ $<0.001$ por PCS) fueron medianos. El tamaño de efecto directo del entorno peatonal inmediato sobre el afrontamiento $(\beta=0.27$, IC $95 \%$ : $0.15,0.40, p<$ 0.001 por PCS) y el indirecto sobre el envejecimiento exitoso $(\beta=0.25$, IC $95 \%$ : 0.14, $0.35, p<0.001$ por PCS) fueron pequeños. El tamaño del efecto del afrontamiento sobre el envejecimiento exitoso fue directo y muy grande $(\beta=0.91$, IC $95 \%$ : $0.79,1, p<0.001$ por PCS). Se explicó 82\% de la varianza del envejecimiento exitoso y $67 \%$ de la varianza del afrontamiento (figura 5).
Figura 5. Modelo final con los estímulos como tres variables manifiestas correlacionadas y con dos correlaciones entre residuos de medida de afrontamiento y envejecimiento exitoso.

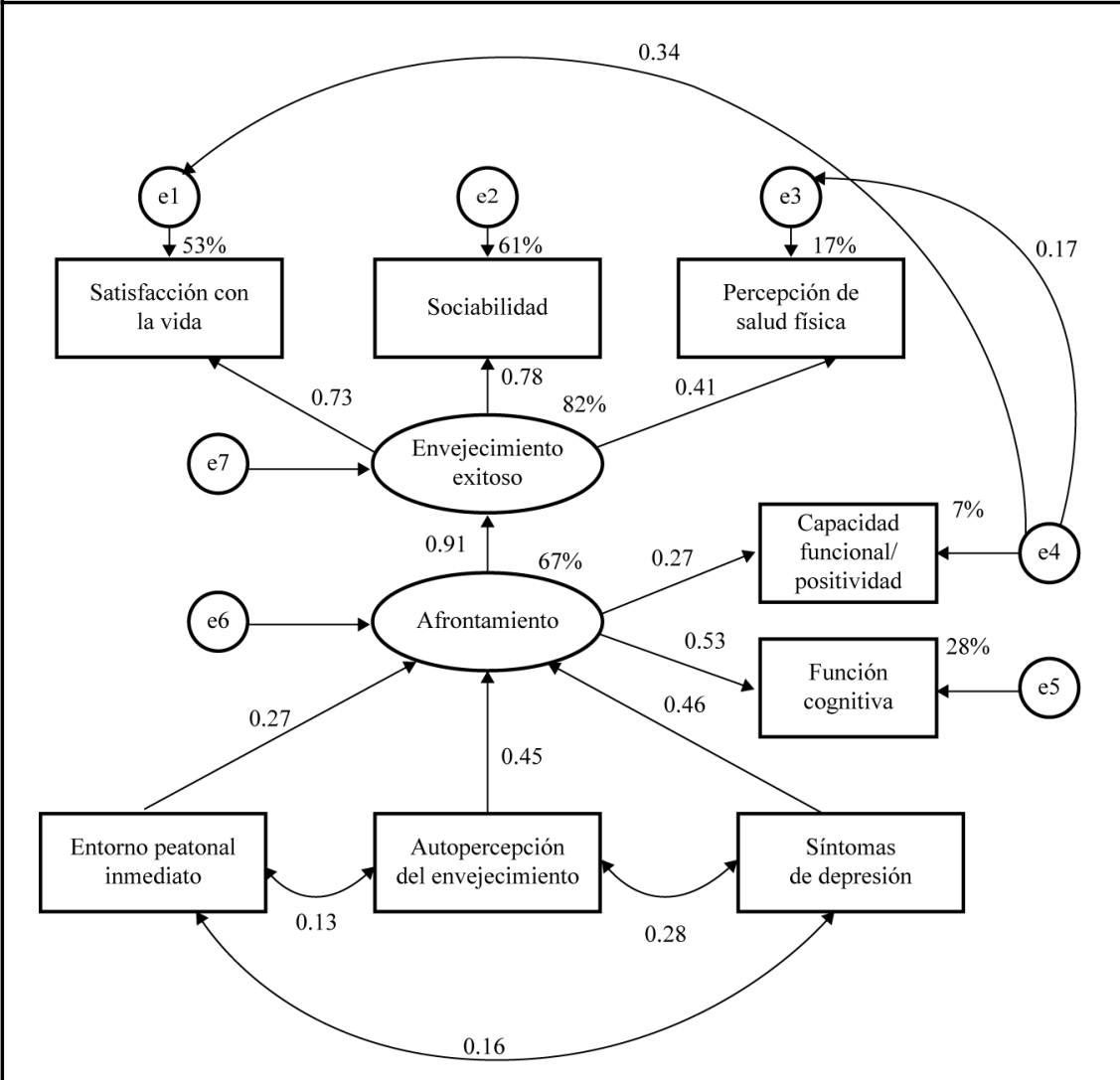

Nota: índices de ajuste: $\chi^{2}(16, N=255)=20.40, p=0.12 ; \mathrm{NFI}=0.95 ; \mathrm{CFI}=0.98 ; \mathrm{SRMR}=0.04 ; \mathrm{RMSEA}=0.04 ; \mathrm{GFI}=$ 0.98 y AGFI $=0.95$.

Fuente: elaboración propia.

\begin{tabular}{|c|c|c|c|c|c|c|c|c|}
\hline Variables & $B$ & $E E$ & $Z$ & $\beta$ & Variables & $S^{2}$ & $E E$ & $Z$ \\
\hline \multicolumn{9}{|c|}{ Modelo estructural } \\
\hline Afr $\leftarrow$ S. D. & 0.08 & 0.02 & $3.51 * * *$ & 0.46 & e6 & 4.12 & 2.16 & $1.74 *$ \\
\hline Afr $\leftarrow$ Ent & 0.16 & 0.05 & $3.03^{* *}$ & 0.27 & & & & \\
\hline Afr $\leftarrow$ Aut & 0.08 & 0.02 & $3.50 * * *$ & 0.45 & & & & \\
\hline Env $\leftarrow$ Afr & 2.79 & 0.68 & $4.08^{* * *}$ & 0.91 & $\mathrm{e} 7$ & 21.26 & 11.36 & 1.87 \\
\hline \multicolumn{9}{|c|}{ Modelos de medida } \\
\hline Sat $\leftarrow$ Env & 1 & & & 0.73 & $\mathrm{e} 1$ & 103.27 & 12.86 & $8.03^{* * *}$ \\
\hline Soc $\leftarrow$ Env & 1.04 & 0.10 & $10.12^{* * *}$ & 0.78 & $\mathrm{e} 2$ & 81.82 & 11.85 & $6.91 * * *$ \\
\hline $\mathrm{Sal} \leftarrow \mathrm{Env}$ & 0.97 & 0.17 & $5.79 * * *$ & 0.41 & $\mathrm{e} 3$ & 543.54 & 50.63 & $10.73^{* * *}$ \\
\hline Aut $\leftarrow$ Afr & 1 & & & 0.27 & $\mathrm{e} 4$ & 158.20 & 14.30 & $11.06^{* * *}$ \\
\hline \multirow[t]{9}{*}{$\mathrm{FC} \leftarrow \mathrm{Afr}$} & 1.47 & 0.41 & $3.57 * * *$ & 0.53 & e5 & 68.07 & 6.75 & $10.08^{* * *}$ \\
\hline & & & & & S. D. & 408.72 & 36.26 & $11.27 * * *$ \\
\hline & & & & & Ent & 35.07 & 3.11 & $11.27 * * *$ \\
\hline & & & & & Aut & 404.38 & 35.88 & $11.27 * * *$ \\
\hline & & & & & S. D.↔Aut & 112.328 & 26.465 & $4.244^{* * *}$ \\
\hline & & & & & S. D.↔Ent & 19.493 & 7.611 & $2.561 * *$ \\
\hline & & & & & Ent $\leftrightarrow$ Aut & 15.326 & 7.534 & $2.034^{*}$ \\
\hline & & & & & $\mathrm{e} 1 \leftrightarrow \mathrm{e} 4$ & 43.615 & 9.785 & $4.457 * * *$ \\
\hline & & & & & $\mathrm{e} 3 \leftrightarrow \mathrm{e} 4$ & 51.280 & 18.309 & $2.801 * *$ \\
\hline
\end{tabular}

Nota: Afr = afrontamiento, Env = envejecimiento exitoso, Sal = percepción de la salud física, Aut = autopercepción del envejecimiento, Soc $=$ sociabilidad, Sat = satisfacción con la vida, Ent = entorno peatonal del vecindario, $\mathrm{S} . \mathrm{D} .=$ síntomas depresivos, $\mathrm{FC}=$ función cognitiva, $e=$ error, $B=$ coeficiente de regresión sin estandarizar, $E E=$ error estándar, $Z=$ prueba de contraste por la $Z$ de Fisher con la hipótesis nula de parámetro igual a cero, $\beta=$ coeficiente de regresión estandarizado, $S^{2}=$ varianza de los residuos o covarianzas. Valor de probabilidad para una prueba $Z$ bilateral: ${ }^{*} p<0.05,{ }^{* *} p<0.01,{ }^{* * *} p<0.001$. Método: máxima verosimilitud.

Fuente: elaboración propia. 


\section{Discusión}

Se planteó que una mayor conciencia o autopercepción del envejecimiento que activa el afrontar las limitaciones y dependencias progresivas, unas valoraciones del entorno peatonal inmediato como facilitador de la deambulación (ambos estímulos focales) y el estímulo contextual de una mayor actividad física a través del caminar, influirían positivamente en los procesos de afrontamiento del envejecimiento. A su vez, el estímulo contextual de menos síntomas de depresión influiría positivamente en los procesos de afrontamiento. Entre los procesos de afrontamiento se incluyó el fomento o preservación de la capacidad funcional, los factores intrapsíquicos de creatividad, baja negatividad, afectividad positiva y control personal, así como una buena función cognitiva (atención, memoria, fluencia verbal, lenguaje y habilidades visuoespaciales). Los son datos concordantes con la hipótesis de manera parcial. La autopercepción del envejecimiento, las valoraciones sobre el entorno peatonal inmediato y los síntomas de depresión tuvieron el efecto esperado, pero no así la actividad física de caminar.

Se concedió una importancia considerable a la actividad de caminar, ya que se pensó que una actividad que realiza diariamente el adulto mayor como ésta sería diferencial entre los adultos mayores físicamente activos o no, en específico en mujeres, en este sentido la práctica de un deporte como trotar o correr quedaría incluida. Un mayor número de pasos (mayor actividad) favorece la circulación y por lo tanto el cerebro estará mejor oxigenado, lo que repercute en un mejor estado físico y función cognitiva (Colcombe y Kramer, 2003; Payette et al., 2010); además, la actividad física y el ejercicio estimulan la producción de endorfinas, lo que impacta de manera positiva en el estado de ánimo (Lubans et al., 2012). Debe señalarse que la mitad de la muestra estuvo en un nivel de sedentarismo (menos de 5000 pasos a la semana) y una cuarta parte de participantes en un nivel de poca actividad (5 000-7 499 pasos), conforme a los criterios de Tudor-Locke et al. (2011), por lo que la actividad física entre estos adultos mayores, que en su mayoría eran mujeres $(83 \%)$ puede calificarse de escasa. La marcada asimetría hacia los valores de baja actividad pudo haber motivado la falta de efecto de esta variable.

Al comparar la media de pasos diarios entre ambos sexos no hubo diferencia estadísticamente significativa, pero cuando se controló la edad los hombres resultaron significativamente más activos que las mujeres. En este sentido, la edad sí estableció diferencia significativa en la actividad de caminar. Considerando la mediana de edad de 68 años, la media de pasos diarios entre los 129 adultos mayores de 60 a 68 años fue significativamente mayor que la de los 126 adultos de más de 68 años. La edad tuvo un tamaño de efecto medio sobre actividad de caminar ( $g$ de Hedges y Olkin $=0.58$, IC $95 \%$ : $0.33,0.84)$. Con la edad, estos adultos mayores se van volviendo más sedentarios; no obstante, no impacta sobre el afrontamiento ni sobre la respuesta de adaptación dentro del modelo especificado. El sedentarismo fue un rasgo general de la muestra.

Los datos parecen reflejar que la distinción entre estímulos focales y contextuales es poco relevante dentro del modelo. Es mejor considerar a las tres variables de estímulos con efecto directo sobre afrontamiento e indirecto sobre envejecimiento exitoso (autopercepción del envejecimiento, entorno peatonal inmediato y síntomas de depresión) como predictores correlacionados. Los tamaños de efecto medianos de los síntomas de depresión y de la autopercepción de envejecimiento comparados con los tamaños de efecto pequeños del entorno peatonal inmediato y el efecto no significativo del caminar reflejan que lo importante para un afrontamiento exitoso entre estos adultos mayores no es estar físicamente activo, sino de modo social, mental y emocional (Paulson et al., 2011).

Se planteó que el afrontamiento con los indicadores de capacidad funcional/positividad y función cognitiva tuviera un efecto positivo sobre el envejecimiento exitoso. Los datos son concordantes con la hipótesis. Reflejan un tamaño de efecto significativo y en la dirección esperada, pero el tamaño de efecto fue muy grande $(\beta=0.91)$. Parece que afrontamiento y envejecimiento exitoso son conceptos muy semejantes al compartir $82 \%$ de la varianza, lo que podría interpretarse como conceptos intercambiables. Cabría probar si con la inclusión de un cuarto indicador para envejecimiento exitoso (una medición específica para la función del rol) y un tercer indicador para afrontamiento (espiritualidad o religiosidad como un factor intrapsíquico) se diferencian más los conceptos. Precisamente Vance et al. (2011) recomiendan indagar por qué los participantes con alta religiosidad experimentan beneficios diferenciales en la salud y perciben mayor apoyo social que quienes reportan baja religiosidad. En el modelo de Roy la espiritualidad se puede contemplar como un factor intrapsíquico (Roy, 2009).

Las dos correcciones entre residuos de medida introducidas en el modelo para lograr un buen ajuste a los datos reflejan que la capacidad funcional/positividad está relacionada con la satisfacción con la vida y la percepción de salud física en aspectos no contemplados en este modelo, probablemente en aspectos afectivos de bienestar y optimismo, ya que el afrontamiento, dentro del modelo, fue indicado sobre todo por un buen funcionamiento cognitivo y en menor medida por la capacidad funcional/positividad para enfrentar demandas físicas, ocupacionales y adaptativas del envejecimiento. 


\section{Conclusiones}

Se concluye que el apoyo de los datos al modelo derivado del modelo de adaptación de Roy es parcial. La actividad física de caminar (estímulo contextual) no tiene efecto sobre el afrontamiento y la distinción entre estímulos focales y contextuales no es relevante dentro del modelo. En concordancia con las hipótesis, los síntomas de depresión, la autopercepción de envejecimiento y los aspectos autoevaluativos de la persona sobre el entorno peatonal en el que viven se relacionan entre sí y activan los procesos de afrontamiento (fomento de la capacidad funcional y la actitud positiva y conservación de la función cognitiva), los cuales a su vez provocan respuestas adaptativas para el ciclo actual de vida de los adultos mayores (satisfacción con la vida, sociabilidad y percepción de salud física).

Como limitación del estudio debe señalarse que el muestreo fue no probabilístico. Se trató de compensar esta limitación eligiendo de forma aleatoria los centros DIF y se tomó la estrategia de tomar muestras proporcionales en cada grupo de reunión en estos centros. Debe señalarse que el sesgo hacia el género femenino que tiene esta muestra se debió a que la población atendida en los servicios médicos de la UANL y centros DIF es mayoritariamente femenina, por lo que los resultados de esta investigación pueden ser aplicados a modo de hipótesis a poblaciones en condiciones similares a la estudiada (1 hombre por cada 5 mujeres). Se optó por el método de la constancia o secuencia fija para agilizar el proceso de aplicación de las escalas. Por tanto, el no contrabalancear la secuencia de las ocho escalas aplicadas para controlar el efecto de la fatiga a la hora de contestarlas constituye otra limitación del estudio, ya que el contrabalanceo es la mejor técnica para control dicho efecto (Shaughnessy et al., 2006).

\section{Sugerencias y prospectiva}

Se recomienda en futuros estudios contemplar la espiritualidad o religiosidad dentro de los indicadores de afrontamiento (factor intrapsíquico) y el modo adaptativo relacionado con el rol (desde el aspecto de integridad del yo versus desesperanza resaltado por Erikson, 2000) entre los indicadores de envejecimiento exitoso con el fin de hacer los conceptos de afrontamiento y envejecimiento exitoso más diferenciables. Además, se sugiere evaluar la actividad física no sólo con los pasos diarios, sino también con la práctica de deportes, como nadar o bailar. Sería importante realizar un contraste multigrupo del modelo entre adultos mayores por grupos de edad. Se sugiere comparar dos grupos de 200 participantes cada uno, usando 70 u 80 años como punto de corte. Asimismo, realizar un contraste multigrupo del modelo entre ambos sexos.

En México, como en el resto de los países latinos, se está incrementando la esperanza de vida y está envejeciendo la población (Hernández et al., 2013). Cabe preguntarse cuál será la evolución de la respuesta de adaptación de los adultos mayores en las próximas décadas a la luz del presente modelo. El modelo muestra que en la medida en que no se experimente depresión, se tiene una conciencia del envejecimiento que activa afrontar las limitaciones y dependencias progresivas y se logra conservar un buen funcionamiento cognitivo; aun en una persona sedentaria (que camine poco), se garantiza un envejecimiento exitoso. Por otra parte, los añadidos al modelo (las dos correlaciones entre residuos de medida) muestran que los rasgos personales de positividad y optimismo contribuyen a un mayor bienestar y percepción de salud. Consecuentemente, en la medida que las políticas públicas se enfoquen hacia un concepto de adulto mayor activo, pensador, crítico, responsable, positivo y autónomo lograrán fomentar el envejecimiento exitoso. Por el contrario, si se enfocan hacia un concepto de adulto pasivo, dependiente e incapaz de pensar, lo dificultarán

Referencias

APA (American Psychological Association). (2002). Ethical principles of psychologists and code of conduct. American Psychologist, 57, 1060-1073. doi:10.1037/0003066X.57.12.1060

Arbuckle, J. L. (2007). AMOS 16.0 user's guide. Spring

House Amos Development.

Barker, M., O’Hanlon, A., McGee, H. M., Hickey, A. y Conroy, R. M. (2007). Cross-sectional validation of the Aging Perceptions Questionnaire: A multidimensional instrument for assessing self-perceptions of aging. $B M C$ Geriatrics, 7, 9. doi:10.1186/1471-2318-7-9

Barrett, A. J. y Murk, P. J. (2006). Life Satisfaction Index for the Third Age (LSITA): A measurement of successful aging, en E. Isaac (ed.), Proceedings of the 2006 Midwest research-to-practice conference in adult, conti- nuing, and community education (pp. 7-12). St. Louis: University of Missouri-St. Louis.

Beard, J. R. y Petitot, C. (2010). Ageing and urbanization: Can cities be designed to foster active ageing? Public Health Reviews, 32, 427-450. Cerin, E., Saelens, B. E., Sallis, J. F. y Frank, L. D. (2006). Neighborhood Environment Walkability Scale: Validity and development of a short form. Medicine and Science in Sports 
and Exercise, 38, 1682-1691. doi: 10.1249/01. mss.0000227639.83607.4d

Chodosh, J., Kado, D. M., Seeman, T. E. y Karlamangla, A. S. (2007). Depressive symptoms as a predictor of cognitive decline: MacArthur studies of successful aging. The American Journal of Geriatric Psychiatry, 15, 406-415. doi:10.1097/01.JGP.0b013e31802c0c63

Colcombe, S. y Kramer, A. F. (2003). Fitness effects on the cognitive function of older adults: A meta-analytic study. Psychological Science, 14(2), 125-130. doi:10.1111/14679280.t01-1-01430

Conversano, C., Rotondo, A., Lensi, E., Della Vista, O., Arpone, F. y Reda, M. A. (2010). Optimism and its impact on mental and physical well-being. Clinical Practice and Epidemiology in Mental Health, 6, 25-29. doi:10.2174/1745017901006010025

Crouter, S. E., Schneider, P. L., Karabulut, M. y Bassett, D. R. (2003). Validity of 10 electronic pedometers for measuring steps, distance, and energy cost. Medicine and Science in Sports and Exercise, 35, 1455-1460. doi:10.1249/01. MSS.0000078932.61440.A2

Deng, J., Hu, J., Wu, W., Dong, B. y Wu, H. (2010). Subjective well-being, social support, and age-related functioning among the very old in China. International Journal of Geriatric Psychiatriy, 25, 697-703. doi:101002/gps.2410.

Dogra, S. y Stathokostas, L. (2012). Sedentary behavior and physical activity are independent predictors of successful aging in middle-aged and older adults. Journal of Aging Research doi:10.1155/2012/190654

Durán, D., Orbegoz, L. J., Uribe-Rodríguez, A. F. y Uribe, J. M. (2008). Integración social y habilidades funcionales en adultos mayores. Universitas Psychologica Panamerican Journal of Psychology, 7(1), 263-270.

Erikson, E. (2000). El ciclo vital completado. Edición revisada y ampliada. Barcelona: Paidós.

Flood, M. (2005). A mid-range nursing theory of successful aging. Journal of Theory Construction \& Testing, 9(2), 35-39.

Hernández, M. F., López, R. y Velarde, S. I. (2013). La situación demográfica en México. Panorama desde las proyecciones de población. Ciudad de México: Conapo.
Herrero, J. y Gracia, E. (2007). Una medida breve de la sintomatología depresiva. Salud Mental, 30(5), 40-46.

Kantún-Marín, M. A., Moral de la Rubia, J. Gómez-Meza, M. V. y Salazar-González, B. C. (2013). Validación del índice de satisfacción con la vida en la tercera edad. Aquichan, 13(2), 148-158.

Kleinspehn, A., Kotter, D. y Smith, J. (2008). Self-perceptions of aging: Do subjective age and satisfaction with aging change during old age? Journal of Gerontology, 63, 377-385. doi:10.1093/geronb/63.6

Lee, P., Lau, W. y Yen, T. W. (2011). Aging successfully: a four-factor model. Educational Gerontology, 37, 210-227. doi:10.1080/03601 277.2010.487759.

Lubans, D. R., Plotnikoff, R. C. y Lubans, N. J. (2012). Review: A systematic review of the impact of physical activity programmes on social and emotional well-being in at-risk youth. Child and Adolescent Mental Health, 17(1), 2-13. doi:10.1111/j.1475-3588.2011.00623.x

Mathuranath, P. S., Nestor, P. J., Berrios, G. E., Rakowicz, W. y Hodges, J. R. (2000). A brief cognitive test battery to differentiate Alzheimer's disease and frontotemporal dementia. Neurology, 55, 1613-1620. doi:10.1212/01.wnl.0000434309.85312.19

Miodek, A., Szemraj, P., Kocur, J. y Ryś, A. (2007). Masked depression-History and present days. Polski Merkuriusz Lekarski, 23(133), 78-80.

Ng, T. P., Broekman, B. F., Niti, M., Gwee, X. y Kua, E. H. (2009). Determinants of successful aging using a multidimensional definition among Chinese elderly in Singapore. The American Journal of Geriatric Psychiatry, 17, 407416. doi:10.1097/JGP.0b013e31819a808e

Paulson, D., Bowen, M. E. y Lichtenberg, P. A. (2011). Successful aging and longevity in older old women: the role of depression and cognition. Journal of Aging Research.

Payette, H., Gaudreau, P., Morais, J. A., Shatenstein, B. y Gray-Donald, K. (2010). Trajectories of physical function decline and psychological functioning: the Quebec longitudinal study on nutrition and successful aging (NuAge). The Journals of Gerontology Series B: Psychological Sciences and Social Sciences,
66(Suppl. 1), doi:10.1093/geronb/gbq085. Roy, C. y Andrews, H. (1999). The Roy adaptation model (2a ed.). Stanford: Appleton \& Lange. Roy, C. (2009). The Roy adaptation model (3a ed.). Upper Saddle River: Pearson Education.

Rubio, R. y Aleixandre, M. (1999). La escala Este, un indicador objetivo de soledad en la tercera edad. Geriátrika. Revista Iberoamericana de Geriatría y Gerontología, 15(9), 26-35.

Salinas, A., Manrique, B., Acosta, G. I., Franco, A., Rosas, Ó., Gutiérrez, L. M. y Sosa, A. L. (2014). Validación de un punto de corte para la versión breve de la Escala de Depresión del Centro de Estudios Epidemiológicos en adultos mayores mexicanos. Salud Pública de México, 56(3), 279-285.

Shaughnessy, J. J., Zechmeister, E. B. y Zechmeister, J. S. (2006). Research methods in psychology. New York: McGraw-Hill.

Troutman, M., Nies, M. A., Small, S. y Bates, A. (2011). The development and testing of an instrument to measure successful aging. Research in Gerontological Nursing, 4(3), 221-232. doi:10.3928/19404921-20110106-02

Tudor-Locke, C., Craig, C. L., Brown, W. J., Clemes, S. A., De Cocker, K., Giles-Corti, B., Hatano, Y., Ivone, S., Matsudo, S. M., Nutrie, N., Oppert, J. M., Rowe. D. A., Schmidt, M. D., Schofield, G. M., Spence, J. C., Teixeira, P. J., Tully, M. A., Blair, S. N. (2011). How many steps/day are enough? for adults. The International Journal of Behavioral Nutrition and Physical Activity, 8, 79. doi:10.1186/1479-5868-8-79.

Vance, D. E., Brennan, M., Enah, C., Smith, G. L. y Kaur, J. (2011). Religion, spirituality, and older adults with HIV: critical personal and social resources for an aging epidemic. Clinical Interventions in Aging, 6, 101-109. doi:10.2147/ CIA.S16349

Ware Jr, J. E., Kosinski, M. y Keller, S. D. (1996). A 12-Item Short-Form Health Survey: construction of scales and preliminary tests of reliability and validity. Medical Care, 34, 220-233. doi:10.1097/00005650-199603000-00003

WHO (World Health Organization) (1992). Activities of WHO 1990-1991: Biennial report of the general director of the World Health Assembly and the United Nations. Geneva: World Health Organization. 